\title{
Simple and Rapid Detection of Burkholderia and Vari- olla Using Multiplex-PCR
}

\author{
Mona Simkhah $^{1}$ (D), Mohammad Javad Dehghan Esmatabadi ${ }^{*}$ (D, Mehdi Zeinoddini' ${ }^{1}$ (D), Nafiseh Pourmahdi ${ }^{1}$ (D)
}

1. Faculty of Chemistry and Chemical Engineering, Malek Ashtar University of Technology, Iran.

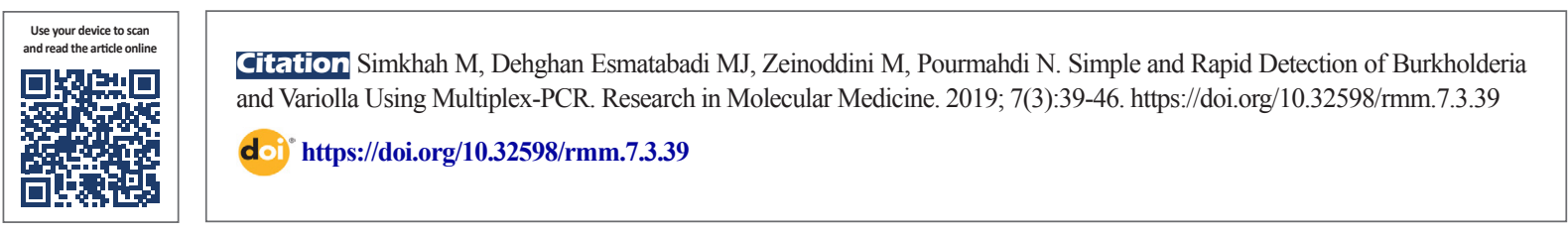

\section{(i) (5)}

Article Type:

Research Paper

Article info:

Received: 29 Jun 2019

Revised: 23 Jul 2019

Accepted: 14 Aug 2019

Keywords:

Hybrid construct, Positive control sample, PCR, Burkholderia, Variolla

\begin{abstract}
A B S T RA C T
Background: Todays, one of the most important problems in detection of human pathogens, is lack of positive control. The idea of using hybrid vectors, containing genes of different pathogens, can overcome this limitation. We can design specific primers for each region and use the hybrid vector as positive control sample in PCR. In this research we designed a hybrid vector and relevant primers for detection of Variolla and Burkholderia.

Materials and Methods: In this study 16srRNA and HA genes were chosen to be located on the vector, to represent of Burkholderia and Variolla, in respectively. The sequence of these genes obtained from NCBI in FASTA format and aligned in BioEdit software for finding conserve region of each gene, then some purposeful changes were applied in the sequence of each gene and the sequences were placed next to each other and the construct was designed. Specific primers designed for each region using Oligo7, BioEdit, GeneRunner softwares, Oligo analyzer website and NCBI database. Finally, the construct cloned in PUC57 in SnapGene and PCRsimulated on hybrid vector using designed primers.

Results: Analysis confirmed that conserved region for each gene is located on hybrid vector for each pathogen, and simulation of PCR proved the accuracy of designed primers.

Conclusion: Hybrid vectors design contain similar sequence of pathogens genome but they are nonepathogenic. We can use these hybrid vectors as positive control, without any concern.
\end{abstract}

\section{Introduction}

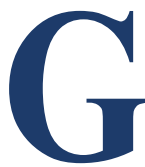

enetic vectors are engineered and artificial tools used in molecular cloning. A genetic vector contains an external genetic sequence is a recombinant vector. The recombinant or hybrid vectors are tools for transmitting, reproducing or expressing several different genetic components simultaneously in one or more specific hosts [1]. The ability of carrying different genetic sequences in hybrid vectors, lead to using them as positive control sample for detection of some pathogens. These hybrid vectors are cloned in a host and can be used when required. One of the most important hosts is Escherichia coli, which is used for keeping and replicating of hybrid vectors [2]. Centers for Disease Control

\footnotetext{
* Corresponding Author:

Mohammad Javad Dehghan Esmatabadi, PhD.

Address: Faculty of Chemistry and Chemical Engineering, Malek Ashtar University of Technology, Iran.

Phone: +98 (912) 4765135

E-mail:mohammad_dehghan@mut.ac.ir
} 
and Prevention(CDC) classify biological agents in category $\mathrm{A}, \mathrm{B}$ and $\mathrm{C}$ for prevention actions [3].

Laboratories are classified in 4 biosafety level according to their equipment, so working on dangerous pathogens require equipped lab [4]. Biological agents usually spread quickly and have high mortality rate, so rapid detection of these pathogens are valuable [5]. Culture and immunological base detection methods are costly and time consuming, but molecular detection methods are quick and reliable $[2,3]$. There are some limitations for molecular detection of biological agents, such as, 1 . accessing to the genome of pathogens to use as positive control sample is difficult. 2. In order to ensure that these factors are not present, we always have to test several microorganisms simultaneously using different controls [4]. 3.The presence of false positive results due to contamination of the positive control is problematic. 4 . The level of existing laboratory equipment and existing methods in clinical laboratories and the lack of access to common commercial tests is a major limitation [5].

Using simulation techniques is a good idea to solve many problems associated with threatening biological agents, but very few simulators have been identified so far. In biological defense, a few simulators, including Bacillus subtilis (as an alternative to Bacillus anthracis), Panto aglomerats (as an alternative to all threaten plants bacteria), and Phage M13 (as an alternative to all viral threats), are used, But these simulators are ineffective in molecular methods because they have no common gene with the real pathogen,so design and construction of hybrid vectors seems to be more efficient [6]. Hybrid vectors have similar genetic region of the biological agent, but when they applied instead of these pathogens, they eleminate all the problems and deficiencies mentioned above; hence, these hybrid vectors act as a kind of genetic simulators. In fact, this strategy is the application of positive control sequence is in order to confirm the presence of a specific organism in a sample.

In this research, we designed a kind of vector consisting different genetic units of specific microbial or viral agents and can be used as a positive control in the PCR process by designing specific primers for the same genetic unit and bycomparing the amplified fragment from vector and pathogen. So, based on the existing priorities in Iran, biological agents including Burkholderia mallei and Pseudomallei and Variolla were selected to be placed in the hybrid vector [7-16]. On the other hand, due to the many advantages of Multiplex PCR, we have designed the vector and primers, based on a multiple PCR reaction, in order to be able to use this information a diagnostic kit of four pathogens, based on the PCR process.

\section{Materials and Methods}

\section{Selection of conserved gene for each pathogen:}

In the first step, in order to improve the accuracy of detection, a specific genetic sequence must be selected for each species. This sequence must be so specific and it does not exist in other organisms, especially do not exist even in other none-pathogenic species or strains that can differentiate between them. In order to find conserved genes, of two mentioned pathogens, numerous articles were evaluated from NCBI and the INSDC databases (The International Nucleotide Sequence Database Collaboration). Conserved genes for the pathogens of Burkholderia mallei and Pseudomallei and Variolla were 16srRNA, the component of the 30S small subunit of a prokaryotic ribosome that binds to the Shine-Dalgarno sequence $[6,7]$ and HA (Hemagglutinin protein), an infected-cell membrane antigen [8-10], Respectively.

\section{Sequence for each gene:}

The NCBI database was used to prepare the sequence of genes. This database is the center for information about genes, their transcriptions, proteins, and many possibilities for biosciences [17]. In this research, all sequences related to the two identified genes for all species and subtypes of these pathogens were extracted from the database and stored in the FASTA format.

Table 1. Designed primers and their characteristics

\begin{tabular}{|c|c|c|c|c|c|c|c|c|}
\hline Pathogen & $\begin{array}{l}\text { Target } \\
\text { Region }\end{array}$ & & Primer Sequence & $\begin{array}{l}\text { Primer } \\
\text { Length }\end{array}$ & $\mathrm{Tm}$ & GC\% & $\begin{array}{l}\text { Product Size } \\
\text { In Construct }\end{array}$ & $\begin{array}{l}\text { Product Size in Origi- } \\
\text { nal Agents }\end{array}$ \\
\hline \multirow{2}{*}{ Variolla } & \multirow{2}{*}{$\mathrm{HA}$} & $\mathrm{F}$ & 5'- AATCCACAACAGACAAGACGT & 21 & 57.8 & 42.9 & \multirow{2}{*}{$333 b p$} & \multirow{2}{*}{$233 b p$} \\
\hline & & $\mathrm{R}$ & 5'- ACCAAATACTTTGACATAGTC & 21 & 51.2 & 32.3 & & \\
\hline \multirow{2}{*}{ Burkholderia } & \multirow{2}{*}{ 16srRNA } & $\mathrm{F}$ & 5'-TTCTGGCTAATACCCGGACTG & 21 & 59.2 & 52.4 & \multirow{2}{*}{$438 \mathrm{bp}$} & \multirow{2}{*}{$338 \mathrm{bp}$} \\
\hline & & $\mathrm{R}$ & 5'-TATCTAATCCTGTTTGCTCC & 20 & 52 & 42.9 & & \\
\hline
\end{tabular}




\section{Conserve region of each gene:}

BioEdit software was used to determine the conserveregion of each gene. BioEdit is a powerful software for sequence alignment which use the ClustalW Multiple Alignment method [18]. In this research, obtained sequences in the previous stage were aligned with this software and the conserved region of each gene were determined to be placed on the final construct.

\section{Primer design}

OLIGO software version 7 was used to design the primer. OLIGO is an essential software for designing and analyzing PCR primers. At this stage, the conserve region of each gene transmitted to the OLIGO software and forward and reverse primers were designed for each gene, and finally, the properties of primers including of dimer, binding temperature, CG percentage, and PCR product length were evaluated. Designed primers and their characteristics are listed in Table 1.

\section{Specifity of primer}

At this stage, NCBI Primer Blast website was used to determine the specificity of each primer by selecting NR databank (just to identify the specific agent), and the specificity of the primers was evaluated.

\section{Analysis of primers for Multipelex PCR}

In the first step, the online website of Oligo Analyser was used to analyse the primers.After designing primers, dimer formation and stability of primers were studied and suitable primers were designed. In the next step, primers were analysed in the NCBI PrimerBlast website by selecting NR databank, in which the primers of eachpathogen were examined two to two, with the aim of not identifying any pathogen, and finallyall primers were confirmed.

\section{Hybrid construction design}

At this stage, using the creative idea of the researches of Miss. Monica Carrera and et al [11, 12] certain changes applied in target gene region of each pathogenin order to be placed in the construction, which is expressed below:

1- A sequence with a length of $100 \mathrm{bp}$ with EcoRI and NotI restriction site was considered for inside of each gene. This $100 \mathrm{bp}$ sequence was added because of two reason: First, if the specimen containing the pathogen is negligently contaminated with a very small amount of synthetic positive genetic control, in that case, we can use the enzymatic digestion method to differentiate these two from each other. So, by performing enzymatic digestion operations on the suspect contaminated sample, all of the synthetic genetic positive controls will be broken down and, as a result, we will not have false positive and negative results. Second, providing difference in size for each agent on the structure and original sample, so, if restriction enzymes are not performed, it will provide a definite answer with the aid of this difference in size on the agarose gel.

2- A sequence of $48 \mathrm{bp}$ was considered for between sequences with XhoI and BamHI restriction sites. The enzymatic pre-treatment before PCR prevents the production of unwanted parts and also lead to amplification of each region by its own specific primers.

After applying the above changes and placing the sequences together, a 2023 nucleotide sequence was created for placement in the hybrid vector.

\section{Hybrid vector preparation}

SnapGene software was used to design a hybrid vector. As already mentioned, the SacI enzyme restriction site was placed on both sides of the structure. Designed hybrid structure cloned into PUC57 plasmid from SacI restriction site and hybrid vector was simulate.

Structural approval by simulation of the PCR reaction

In order to confirm the design, PCR reaction was simulated in SnapGene software and the agarose gel produced.

\section{Results}

Choosing the best protected gene for each pathogen

Based on the studies and articles, the conserved genes for the Burkholderia mallei and pseudomallei and Variolla were 16 srRNA and HA, respectively.

Obtain sequences for each gene:

All sequences related to the four above genes were extracted and stored in the FASTA format (Figure 1)

\section{Detecting conserved region of each gene:}

Sequences in the previous step were aligned and the conserved region of each gene were determined to be placed on the final structure (Figure 2). 


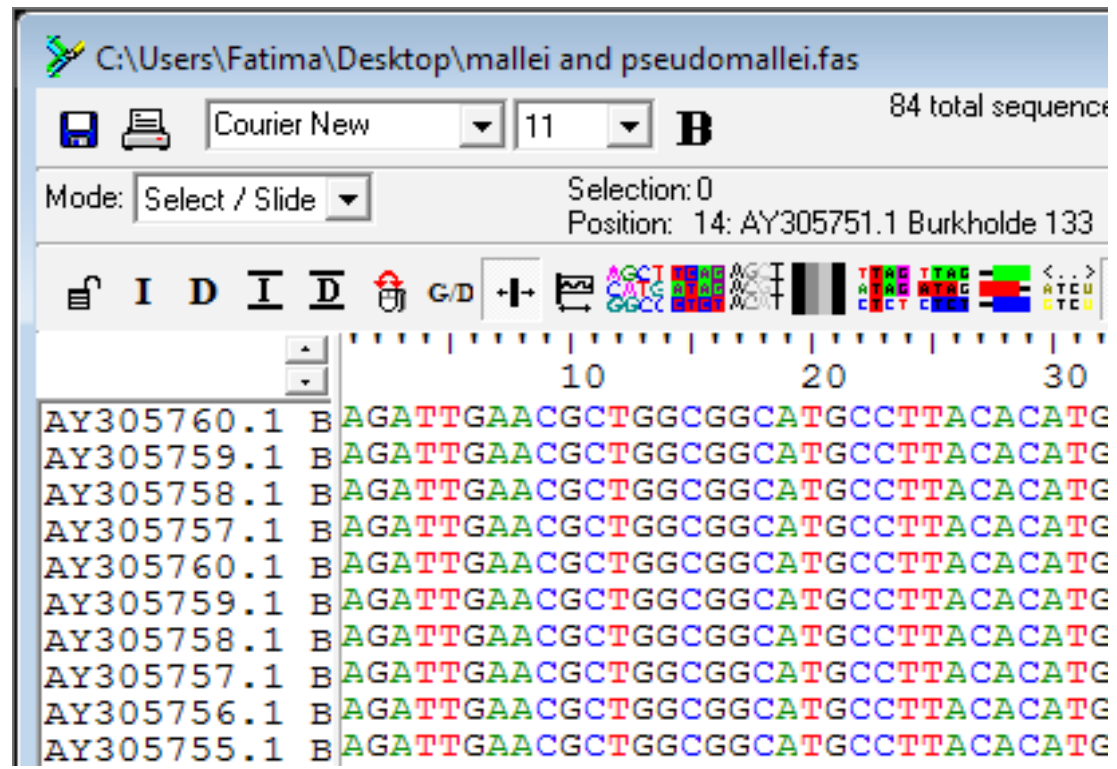

Figure 1. Sequences of 16S ribosomal RNA gene in FASTA format for Borkholderia mallei and pseudomallei for example

Design primer

In the Oligo7 software, a set of different states of primers was randomly evaluated for designing the optimal primers in terms of the absence of dimer primer, CG percentage, stability, Tm and (Figure 3).

\section{Hybrid construction design}

At this stage, certain changes were made in the target gene to be placed in the construct and after placing the sequences together, and final sequence was created for placement in the hybrid vector (Figure 4).

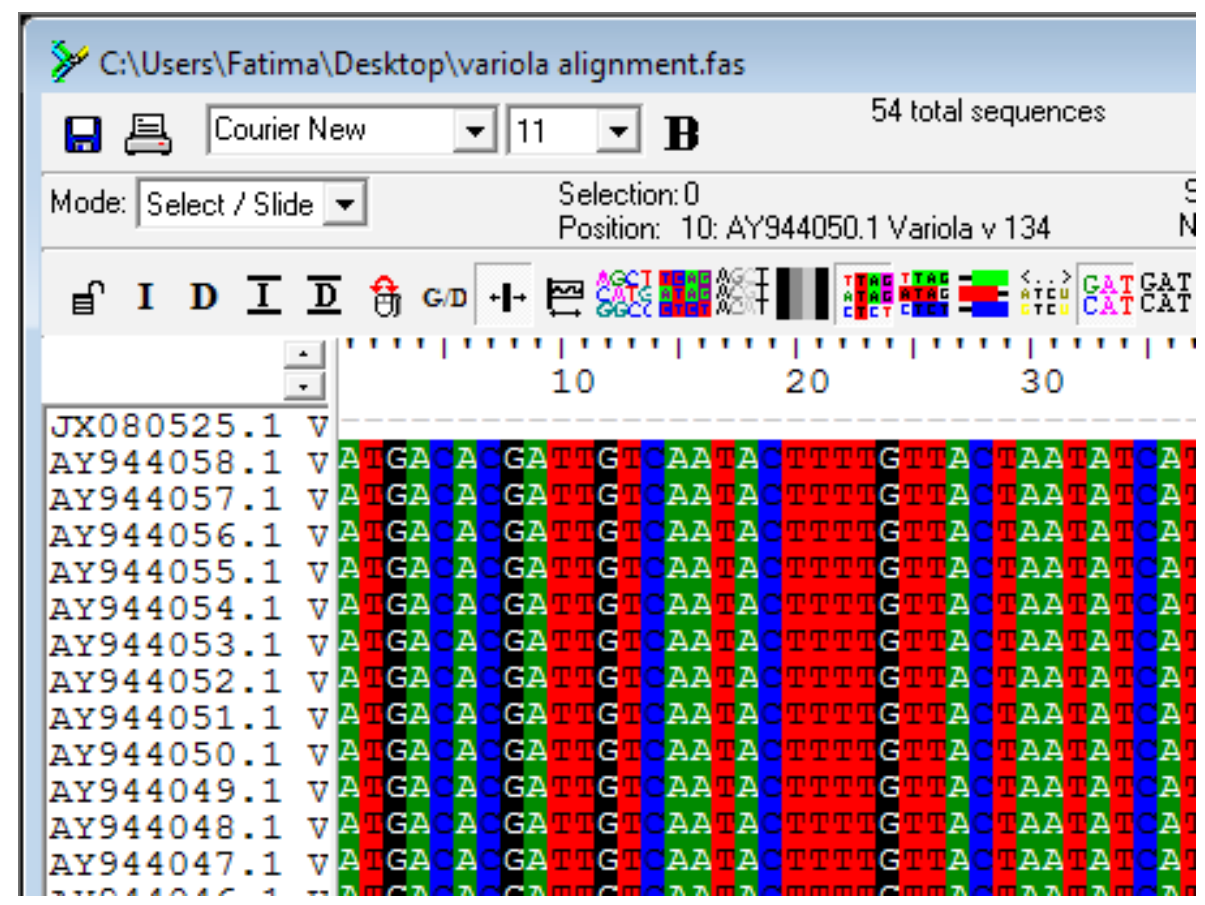

Figure 2. The ClustalW Multiple Alignment process in the BioEdit software for variolla agent sequences for example. 


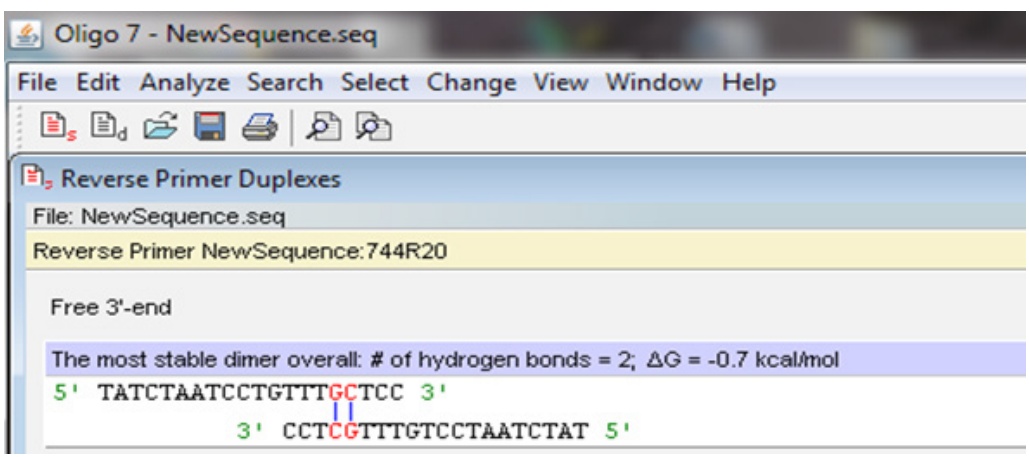

Figure 3. analysis of Burkholderia primer for example

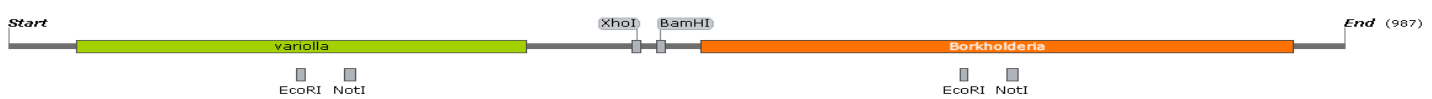

$\underset{9 \times 5}{\operatorname{Lin} \mathrm{bP}}$

Figure 4. Construct schematic: Left to right: Sequence of Variolla (green) with two restriction site EcoRI and NotI - 100bp sequence with two restriction site of XhoI and BamHI -Sequence of Burkholderia (red) with two restriction site EcoRI and NotI.

\section{Hybrid vector simulation}

The construction cloned in PUC57 plasmid, finally hybrid vector was produced (Figure 5). Structural approval by simulation of the PCR reaction:

The PCR reaction was simulated in SnapGene software and results were observed on $2 \%$ agarose gel and expect- ed bands were observed. For the pathogens Burkholderia mallei and Pseudomallei and Variolla, band in the size 333 and 438 bp were expected, respectively (Figure 6).

\section{Specificity of primer Confirmation}

As you can see, when primers were evaluated on the NCBI database on Primer Blast, we identified only one

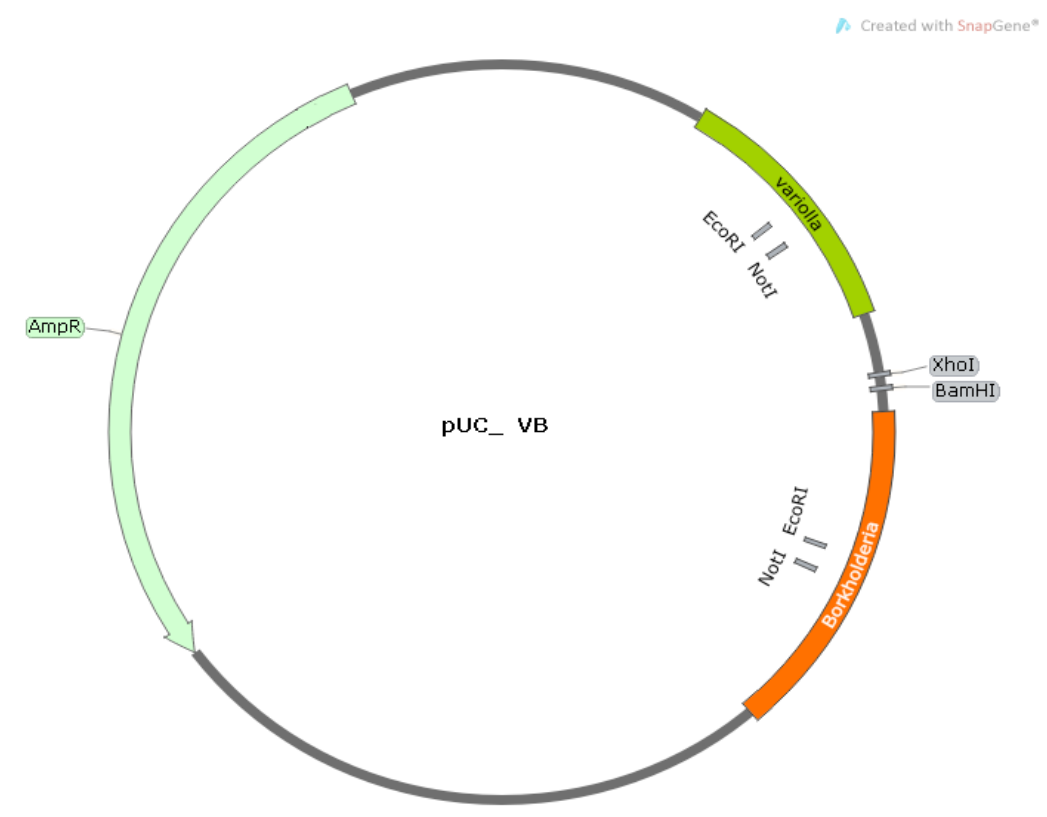

Figure 5. construct schematic: 100bp Sequence with two restriction site of XhoI and BamHI-Sequence of Variolla (green) with two restriction site EcoRI and NotI - 100bp sequence with two restriction site of XhoI and BamHI -Sequence of Burkholderia (red) with two restriction site EcoRI and NotI. 


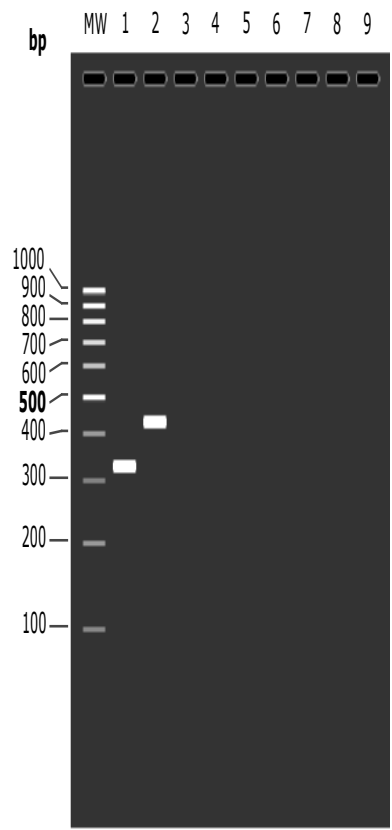

\section{grmm}

Figure 6. Simulation of agarose gel (molecular marker bp 100)

1. Varoilla (333 bp); 2. Burkholderia (438bp)

pathogen for each primer, and no nonspecific product was found; thus, the $100 \%$ specificity of the design primers was confirmed (Figure 7).

\section{Proof of specificity with 4 pairs of primers}

To determine that in the multiplex PCR, 4 primers just determine only 2 designed genetic sequences and do not produce nonspecific pieces (100\% specificity); these 4 primers are 2 to 2 randomly (32 modes) on the PrimerBlast NCBI database analyzed (Table 2) and showed that 2 pairs of primers in a multiplex state are certainly specific similar to monoplex state.

\section{Disscussion}

It's been years, instead of using a real biological threat, such as Burkholderia mallei and Pseudomallei and Variolla, simulators are used as a genetic positive control, because the genetic simulator and main pathogen have similar properties (similar nucleic acid sequences), but the genetic simulator is non-pathogenic.

This type of hybrid structure can effectively replace withreal pathogen. One of the main barriers for designing new kits based on the PCR process for biological agents is the lack of a positive genetic control. Positive control sample is neccessary for testing the performance of a diagnostic kit (including primers or probes). Consequently, the production of this kind of genetic positive control is considered as the research priority of the country's biological defense. Therefore, for this purpose, we need to: 1- In Silico design of construct. 2-test the effectiveness of primers and optimize them in the PCR process on the construct. On the other hand, because of the great advantages of Multiplex PCR, it is better to design structures and primers for a multiplex PCR reaction then we can produce a multiple diagnostic kit based on the PCR process. Studies around the world, shows very few documents, and it seems that only 2 studies based on such a subject which is published as patents and articles.

In a study in France in 2004, these hybrid vectors were used to provide a kind of positive control to overcome many problems that were encountered in detecting pathogens. In this experiment, two different plasmids were designed as a positive control which contained 4 and 6 genetic sequences of dangerous pathogens. They also designed primers and probes to detect each piece, either on the positive control plasmid and the main

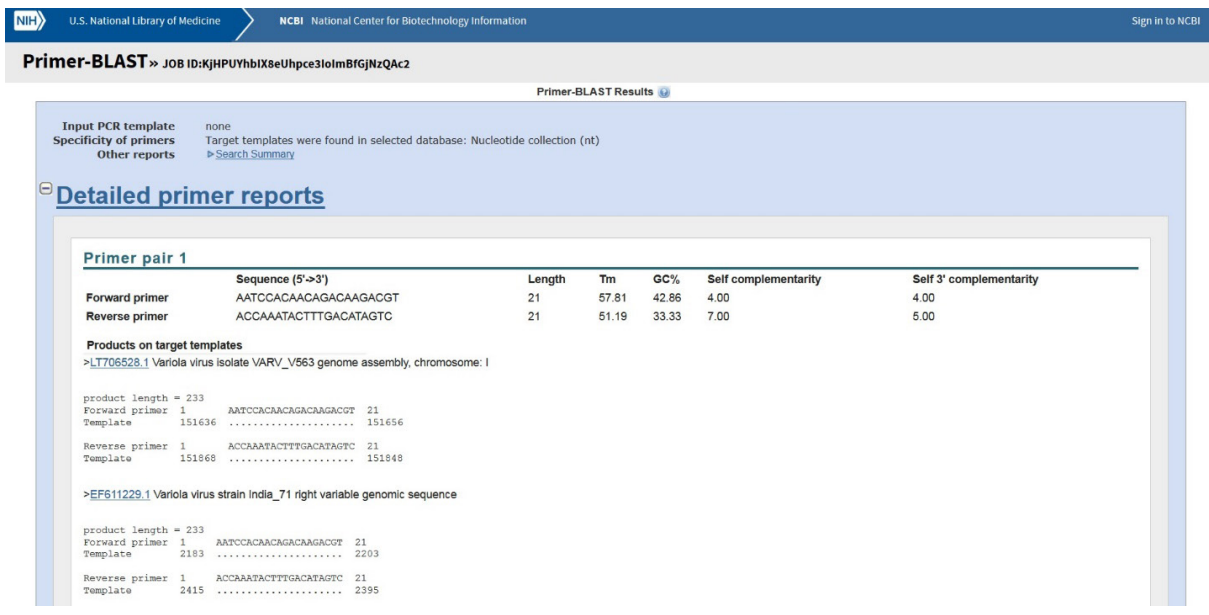

Figure 7. Confirmation of the specificity of the pair of primers in PrimerBlast, for example: Variolla 
Table 2. Specificity of 4 pairs of primers in two to two: Variolla (F1 and R1); Burkholderia (F2 and R2)

\begin{tabular}{|c|c|c|c|c|c|c|c|c|c|}
\hline Type & F1 & F1 & F1 & F2 & R1 & R1 & R2 & F2 & F1 \\
\hline Iуре & F1 & R1 & F2 & F2 & R1 & R2 & R2 & R2 & R2 \\
\hline Result & - & + & - & - & - & - & - & + & - \\
\hline Product length & - & 233 & - & - & - & - & - & 338 & - \\
\hline
\end{tabular}

pathogen. In this test, 8 nucleotides of the main sequence were changed with the 20 nucleotide that contained the restriction site of the NotI [15-19].

Other study, is two patents in the United States, which were published in 2008. They designed a simulator that detect 20 pathogens simultaneously. In this research, BamHI restriction site was located between the sequences of each pathogen [20-23].

In another study in Iran, Zeinoddini et al., in 2019, used a chimeric construct for simultaneous detection of Francisella and Yersinia in a multiplex PCR method. In this study, the fopA gene from Francisella and the cafl gene from Yersiniawas selected, which produced 107and 176 nucleotides, respectively [24].

In two studies in the Pasteur Institute of Iran on Francisella and Yersinia, a hybrid structure was designed and used as a monoplex PCR positive control $[25,26]$.

In this project, a hybrid vector with corresponding primers was designed In Silico, for developing Multiplex PCR process to detect Burkholderia mallei and Pseudomallei and Variolla. This can be used as positive control in molecular detection methods.

\section{Ethical Considerations}

Compliance with ethical guidelines

All ethical principles were considered in this article.

Funding

This research did not receive any specific grant from funding agencies in the public, commercial, or not-forprofit sectors.

Authors contribution's

All authors contributed in preparing this article.

\section{Conflict of interest}

The authors declared no conflict of interest.

\section{Acknowledgements}

We appreciate the effective cooperation of the staff and students of the Institute of Biological of Malek Ashtar University of Technology is appreciated and appreciated for all their efforts and contributions during this project. This study was supported by Malek Ashtar University of Technology.

\section{References}

[1] Brown T. Vectors for gene cloning: Plasmids and bacteriophages. In: Brown T. Gene Cloning and DNA Analysis: An Introduction, $6^{\text {th }}$ ed. Hoboken: Wiley-Blackwell; 2010.

[2] Janse I, Hamidjaja RA, Bok JM, van Rotterdam BJ. Reliable detection of Bacillus anthracis, Francisella tularensis and Yersinia pestis by using multiplex qPCR including internal controls for nucleic acid extraction and amplification. BMC microbiology. 2010; 10(1):314. [DOI:10.1186/1471-2180-10314] [PMID] [PMCID]

[3] Kostylev M, Otwell AE, Richardson RE, Suzuki Y. Cloning should be simple: Escherichia coli DH5a-mediated assembly of multiple DNA fragments with short end homologies. PLoS One. 2015; 10(9):e0137466. [DOI:10.1371/journal. pone.0137466] [PMID] [PMCID]

[4] Morris S, Kellogg R, Perry S, Meyer RF, Bray DA, Nichelson $\mathrm{D}$, et al. Detecting bio-threat agents: The laboratory response network. Am Soc Microbiol (ASM NEWS). 2003; 69(9).

[5] Nulens E, Voss A. Laboratory diagnosis and biosafety issues of biological warfare agents. Clin Microbiol Infect. 2002 8(8):455-66. [DOI:10.1046/j.1469-0691.2002.00528.x] [PMID]

[6] Inglesby TV, Henderson DA, Bartlett JG, Ascher MS, Eitzen E, Friedlander AM, et al. Anthrax as a biological weapon: Medical and public health management. JAMA. 1999; 281(18):1735-45. [DOI:10.1001/jama.281.18.1735] [PMID]

[7] Payne DA, Petersen J. Rapid molecular testing for bioterrorism agents: Targets, tactics, and technology. J Clin Ligand Assay. 2002; 25(4):348-57.

[8] Sagripanti J-LaMC. Non-infectious chimeras that include the nucleic acid signature of most bacterial and viral biological 
threat agents; for use in nucleic acid-based biodetectors and diagnostic products for biodefense. Google Patents. 2010.

[9] Allen L-AH. Mechanisms of pathogenesis: Evasion of killing by polymorphonuclear leukocytes. Microbes Infect. 2003; 5(14):1329-35. [DOI:10.1016/j.micinf.2003.09.011] [PMID]

[10] Cheng AC, Currie BJ. Melioidosis: Epidemiology, pathophysiology, and Management. Clin Microbiol Rev. 2007; 20(3):533. [DOI:10.1128/CMR.00018-07] [PMCID]

[11] Dance DAB. Melioidosis and glanders as possible biological weapons. Bioterrorism and infectious agents: A new dilemma for the $21^{\text {st }}$ century. Springer. 2009; 99-145. [DOI:10.1007/978-1-4419-1266-4_4]

[12] Estrada-De Los Santos P, Vinuesa P, Martínez-Aguilar L, Hirsch AM, Caballero-Mellado J. Phylogenetic analysis of Burkholderia species by multilocus sequence analysis. Curr Microbiol. 2013; 67(1):51-60. [DOI:10.1007/s00284-013-03309] [PMID]

[13] Ray CG, Ryan KJ. Sherris medical microbiology: An introduction to infectious diseases. New York: McGraw-Hill; 2004.

[14] Schrallhammer M, Schweikert M, Vallesi A, Verni F, Petroni G. Detection of a novel subspecies of Francisella noatunensis as endosymbiont of the ciliate Euplotes raikovi. Microb Ecol. 2011; 61(2):455-64. [DOI:10.1007/s00248-010-9772-9] [PMID]

[15] Segonds C, Heulin T, Marty N, Chabanon G. Differentiation of Burkholderia species by PCR-restriction fragment length polymorphism analysis of the 16S rRNA gene and application to cystic fibrosis isolates. J Clin Microbiol. 1999; 37(7):2201-8. [DOI:10.1128/JCM.37.7.2201-2208.1999] [PMID] [PMCID]

[16] Wong-Villarreal A, Caballero-Mellado J. Rapid identification of nitrogen-fixing and legume-nodulating Burkholderia species based on PCR 16S rRNA species-specific oligonucleotides. Syst Appl Microbiol. 2010; 33(1):35-43. [DOI:10.1016/j.syapm.2009.10.004] [PMID]

[17] Aitichou M, Saleh S, Kyusung P, Huggins J, O'Guinn M, Jahrling P, et al. Dual-probe real-time PCR assay for detection of variola or other orthopoxviruses with dried reagents. J Virol Methods. 2008; 153(2):190-5. [DOI:10.1016/j.jviromm et.2008.07.018] [PMID]

[18] Anthony RL, Taylor DL, Daniel RW, Cole JL, McCrumb FR. Studies of Variola Virus and immunity in smallpox: I. Variola Virus hemagglutinins. J Infect Dis. 1970; 121(3):295-302. [DOI:10.1093/infdis/121.3.295] [PMID]

[19] Parekh P, Tang Z, Turner PC, Moyer RW, Tan W. Aptamers recognizing glycosylated hemagglutinin expressed on the surface of vaccinia virus-infected cells. Anal Chem. 2010; 82(20):8642-9. [DOI:10.1021/ac101801j] [PMID] [PMCID]

[20] Coordinators NR. Database resources of the national center for biotechnology information. Nucleic Acids Res. 2016; 45(D1):D12-D7. [DOI:10.1093/nar/gkw1071] [PMID] [PMĐ CID]

[21] Hall T, Biosciences I, Carlsbad C. BioEdit: An important software for molecular biology. GERF Bull Biosci. 2011; 2(1):60-1.
[22] Sagripanti JL, Carrera M. Artificial chimeras engineered to simulate multiple biological threat agents. Google Patents; 2011.

[23] Charrel RN, La Scola B, Raoult D. Multi-pathogens sequence containing plasmids as positive controls for universal detection of potential agents of bioterrorism. BMC microbiol. 2004; 4(1):21. [DOI:10.1186/1471-2180-4-21] [PMID] [PMCID]

[24] Carrera M, Sagripanti JL. Non-infectious plasmid engineered to simulate multiple viral threat agents. J Virol Methods. 2009; 159(1):29-33. [DOI:10.1016/j.jviromet.2009.02.021] [PMID]

[25] Carrera M, Sagripanti JL. Artificial plasmid engineered to simulate multiple biological threat agents. Appl Microbiol Biotechnol. 2009; 81(6):1129-39. [DOI:10.1007/s00253-0081715-8] [PMID]

[26] Sagripanti J-L, Zandomeni MC. Method for simultaneously detecting multiple biological threat agents. Google Patents; 2013. 